\title{
ON UNITARY INVARIANT IDEALS IN THE ALGEBRA OF COMPACT OPERATORS
}

\author{
JÓZSEF V. VARGA
}

(Communicated by John B. Conway)

\begin{abstract}
Lie ideals are constructed, which are ideals in the algebra of compact operators but not ideals in the algebra of bounded operators, thus settling a question of $\mathrm{C}$. K. Fong and $\mathrm{H}$. Radjavi in the negative.
\end{abstract}

\section{INTRODUCTION}

Let $\mathscr{H}$ be an infinite dimensional complex Hilbert space. By operators we shall mean bounded linear transformations of $\mathscr{H}$ into itself. Their algebra is denoted by $\mathscr{B}(\mathscr{H})$, while $\mathscr{K}(\mathscr{H})$ is the algebra of all compact operators. By an ideal we mean a two-sided ideal. We say that $\mathscr{L} \subset \mathscr{B}(\mathscr{H})$ is a Lie ideal in $\mathscr{B}(\mathscr{H})$ if $\mathscr{L}$ is such a linear manifold, that $A \in \mathscr{B}(\mathscr{H})$ and $B \in \mathscr{L}$ imply $A B-B A \in \mathscr{L}$. Let $\mathscr{I}(\mathscr{A})$ and $\mathscr{J}(\mathscr{A})$ denote the ideals of $\mathscr{B}(\mathscr{H})$ and $\mathscr{K}(\mathscr{H})$, respectively, generated by the set $\mathscr{A} \subset \mathscr{K}(\mathscr{H})$. Clearly $\mathscr{I}(\mathscr{A}) \supseteq \mathscr{J}(\mathscr{A})$, but equality does not hold in general. For example if $a<0, \mathscr{I}\left(\operatorname{diag}\left(n^{a}\right)\right) \neq \mathscr{J}\left(\operatorname{diag}\left(n^{a}\right)\right)$ (see [2]). But in the case when $\mathscr{A}$ is countable and $\mathscr{J}(\mathscr{A})$ is a Lie ideal in $\mathscr{B}(\mathscr{H})$, we have $\mathscr{J}(\mathscr{A})=\mathscr{I}(\mathscr{A})$, i.e. $\mathscr{J}(\mathscr{A})$ is an ideal in $\mathscr{B}(\mathscr{H})$ too (see $[3,4$. Example]). The answer to whether $\mathscr{J}(\{A\})=\mathscr{I}(\{A\})$ if $0 \leq A \in \mathscr{K}(\mathscr{H})$ appears in [2] along with the question: Is the above implication true in general, i.e. without any restriction on the cardinality of $\mathscr{A}$ ? In the present paper we discuss the case $\mathscr{A}=\mathscr{U}(A)$ where $0 \leq A \in \mathscr{K}(\mathscr{H})$ and $\mathscr{U}(A)=\left\{U A U^{*}: U^{*}=U^{-1}\right\}$, that is, when $\mathscr{A}$ is the unitary orbit of a positive compact operator. Then $\mathscr{J}(\mathscr{U}(A))$ is a unitary invariant manifold, so by [1, Theorem 1] and [3] it is also a Lie ideal in $\mathscr{B}(\mathscr{H})$ (we refer to [3] for generalization of any result in [1] to the nonseparable case). But it turns out that $\mathscr{J}(\mathscr{U}(A))$ is not an ideal in $\mathscr{B}(\mathscr{H})$ when e.g. $A=\operatorname{diag}\left(n^{-1}\right)$.

Received by the editors November 3, 1988, and in revised form October 18, 1989. 1980 Mathematics Subject Classification (1985 Revision). Primary 47D25, 47B10. 


\section{THE MAIN RESULT}

For a compact operator $A, s_{n}(A)$ denotes the $n$th eigenvalue of the squareroot of $A^{*} A,(n=1,2, \ldots)$ (see [4]). We introduce the following notation:

$$
S_{n}(A)=\sum_{i=1}^{N} s_{i}(A) .
$$

The following lemma is well-known (see e.g. [4, II. Lemma 4.1]):

Lemma 1. If $A \in \mathscr{K}(\mathscr{H})$ and $P$ is an arbitrary orthogonal projection with rank $P \leq n$, then

$$
S_{n}(A) \geq|\operatorname{tr}(P A P)| .
$$

Lemma 2. If $0 \leq A \in \mathscr{H}(\mathscr{H})$ has infinite rank and either $K$ or $L$ is compact, then

$$
s_{n}(K A L)=o\left(s_{n}(A)\right) .
$$

If we also have $\sum s_{n}(A)=\infty$, then

$$
S_{n}(K A L)=o\left(S_{n}(A)\right) .
$$

Proof. For the first statement we refer to [2, Lemma 1]. For the second one we note that $\left\{S_{n}(K A L) / S_{n}(A)\right\}_{n=1}^{\infty}$ is the transform of the zero-sequence $\left\{s_{n}(K A L) / s_{n}(A)\right\}_{n=1}^{\infty}$ with the regular matrix $T=\left[t_{i j}\right]$, where $t_{i j}=s_{j}(A) / S_{i}(A)$ if $j \leq i$ and zero otherwise.

Lemma 3. If $D$ is a positive operator and $P$ and $R$ are finite rank orthogonal projections with $P \leq R$, then

$$
\operatorname{tr}(R D R=\operatorname{tr}(P D P)+\operatorname{tr}((R-P) D(R-P)) \geq \operatorname{tr}(P D P) .
$$

Proof. The equality is obvious, if we choose an orthonormal basis of $\operatorname{ran} P$ and extend it to a basis of $\operatorname{ran} R$ and then to a basis of $\mathscr{H}$. For the inequality we note that $(R-P) D(R-P) \geq 0$.

Now we can prove the following

Theorem. Suppose that $A \in \mathscr{K}(\mathscr{H}), 0 \leq A$,

$$
\sum_{n=1}^{\infty} s_{n}(A)=\infty
$$

and

$$
\liminf _{n \rightarrow \infty} \frac{S_{k n}(A)}{S_{n}(A)}=1
$$

for every positive integer $k$. Then $\mathscr{J}(\mathscr{U}(A)) \neq \mathscr{I}(\{A\})$.

Remarks. It is easy to prove that (2) with $k=2$ implies (2) for every integer $k \geq 1$. Conditions (1) and (2) can be satisfied e.g. by $A=\operatorname{diag}\left(n^{-1}\right)$ because then $S_{n}(A)=\log n+r_{n}$ for some $r_{n} \in[0,1]$ and hence we can even write lim instead of liminf in (2). But, for any nonincreasing sequence $\left\{\alpha_{n}\right\}$ with 
$\alpha_{n} \rightarrow 0$ and $\sum \alpha_{n}=\infty$, we can select a subsequence $\left\{\alpha_{n_{k}}\right\}$, such that $\operatorname{diag}\left(\alpha_{n_{k}}\right)$ satisfies the conditions of the theorem.

We know from [1, Theorem 1] that the unitary invariant linear submanifolds are exactly the Lie ideals in $\mathscr{B}(\mathscr{H})$. So $\mathscr{J}(\mathscr{U}(A))$ in the theorem is such a Lie ideal, which is an ideal in $\mathscr{K}(\mathscr{H})$ but is not an ideal in $\mathscr{B}(\mathscr{H})$ (see the question at the end of [2]). Particularly $\mathscr{J}(\mathscr{U}(A))$ is not countably generated as an ideal of $\mathscr{K}(\mathscr{H})$ (see [3]).

Proof. Let $s_{n}=s_{n}(A)$ and $S_{n}=S_{n}(A)$ for shortness. Let $\left\{e_{n}\right\}_{1}^{\infty}$ be an orthonormal sequence in $\mathscr{H}$ such that $A e_{n}=s_{n} e_{n}$. Fix a strictly increasing sequence $\left\{n_{k}\right\}$ of positive integers, for which

$$
\lim _{k \rightarrow \infty} \frac{S_{N \cdot n_{k}}}{S_{n_{k}}}=1
$$

for every positive integer $N$. The existence of such a sequence follows from (2). By choosing a suitable subsequence if necessary, and calling it $\left\{n_{k}\right\}$, we can assume that

$$
\lim _{k \rightarrow \infty} \frac{S_{n_{k-1}}}{S_{n_{k}}}=0
$$

Clearly (3) remains true. Let $\mathscr{H}_{k}=\operatorname{span}\left(e_{1}, e_{2}, \ldots, e_{n_{k}}\right)$, and let $P_{k}$ be the orthogonal projection of $\mathscr{H}$ onto $\mathscr{H}_{k}$. We need the following lemma:

Lemma 4. Suppose that for the compact operator $B$ we have

$$
\liminf _{k \rightarrow \infty} \frac{S_{n_{k}}(B)}{S_{n_{k}}}=0
$$

Suppose furthermore that there exist positive integers $M, N$, complex numbers $a_{i}$, unitary operators $U_{i}$ and bounded operators $K_{j}, L_{j}$ on $\mathscr{H}(i=$ $1,2, \ldots, N, j=1,2, \ldots, M)$ such that either $K_{j}$ or $L_{j}$ is compact $(j=$ $1,2, \ldots M)$ and

$$
0=B+\sum_{i=1}^{N} a_{i} U_{i} A U_{i}^{*}+\sum_{j=1}^{M} K_{j} A L_{j}
$$

Then $\sum_{i=1}^{N} a_{i}=0$.

Proof. Let $R_{k}$ be the orthogonal projection onto $\operatorname{span}\left(\bigcup_{i=1}^{N} U_{i} \mathscr{H}_{k}\right)$. Multiply both sides of (6) from the left and from the right by $R_{k}$, and take traces:

$$
0=\operatorname{tr}\left(R_{k} B R_{k}\right)+\sum_{i=1}^{N} a_{i} \operatorname{tr}\left(R_{k} U_{i} A U_{i}^{*} R_{k}\right)+\operatorname{tr}\left(\sum_{j=1}^{M} R_{k} K_{j} A L_{j} R_{k}\right) .
$$

Clearly $R_{k} \geq U_{i} P_{k} U_{i}^{*}(k=1,2, \ldots, i=1,2, \ldots, N)$. So we have

$$
\begin{aligned}
S_{n_{k}} & =\operatorname{tr}\left(P_{k} A P_{k}\right) \leq \operatorname{tr}\left(U_{i}^{*} R_{k} U_{i} A U_{i}^{*} R_{k} U_{i}\right) \\
& =\operatorname{tr}\left(R_{k} U_{i} A U_{i}^{*} R_{k}\right) \leq S_{N n_{k}}\left(U_{i} A U_{i}^{*}\right)=S_{N n_{k}} .
\end{aligned}
$$


The first inequality follows from Lemma 3, since $U_{i}^{*} R_{k} U_{i} \geq P_{k}$, and the second one follows from Lemma 1 , because rank $R_{k} \leq N n_{k}$. Consequently we have

$$
\left|\operatorname{tr}\left(R_{k} U_{i} A U_{i}^{*} R_{k}\right)-S_{n_{k}}\right| \leq S_{N n_{k}}-S_{n_{k}} .
$$

Subtract $S_{n_{k}} \sum_{1}^{N} a_{i}$ from both sides of (7):

$$
\begin{aligned}
-\sum_{i=1}^{N} a_{i} S_{n_{k}}= & \operatorname{tr}\left(R_{k} B R_{k}\right)+\sum_{i=1}^{N} a_{i}\left(\operatorname{tr}\left(R_{k} U_{i} A U_{i}^{*} R_{k}\right)-S_{n_{k}}\right) \\
& +\operatorname{tr}\left(\sum_{j=1}^{M} R_{k} K_{j} A L_{j} R_{k}\right) .
\end{aligned}
$$

Using (8) and Lemmas 1 and 2 we get

$$
\begin{aligned}
\left|-\sum_{i=1}^{N} a_{i} S_{n_{k}}\right| & \leq\left|\operatorname{tr}\left(R_{k} B R_{k}\right)\right|+\left(S_{N n_{k}}-S_{n_{k}}\right) \sum_{i=1}^{N}\left|a_{i}\right|+\sum_{j=1}^{M}\left|\operatorname{tr}\left(R_{k} K_{j} A L_{j} R_{k}\right)\right| \\
& \leq S_{N n_{k}}(B)+\left(S_{N n_{k}}-S_{n_{k}}\right) \sum_{i=1}^{N}\left|a_{i}\right|+o\left(S_{N n_{k}}\right) \quad(k \rightarrow \infty) .
\end{aligned}
$$

So

$$
\left|\sum_{i=1}^{N} a_{i}\right| \leq \frac{S_{N n_{k}}(B)}{S_{n_{k}}}+\left(\frac{S_{N n_{k}}}{S_{n_{k}}}-1\right) \sum_{i=1}^{N}\left|a_{i}\right|+\frac{o\left(S_{N n_{k}}\right)}{S_{N n_{k}}} \frac{S_{N n_{k}}}{S_{n_{k}}} .
$$

This holds for every $k$. The third term converges to 0 as $k \rightarrow \infty$ and so does the second term by (3). Taking lim inf of both sides we obtain

$$
\left|\sum_{i=1}^{N} a_{i}\right| \leq \liminf _{k \rightarrow \infty} \frac{S_{N n_{k}}(B)}{S_{n_{k}}} \leq N \liminf _{k \rightarrow \infty} \frac{S_{n_{k}}(B)}{S_{n_{k}}}=0
$$

by (5). This proves Lemma 4 .

Now we return to the proof of the theorem. Let $F=\sum_{k=1}^{\infty}\left(P_{2 k}-P_{2 k-1}\right)$, $P_{0}=0$, and $E=\sum_{k=1}^{\infty}\left(P_{k}-P_{k-1}\right)$. First we put $B=F A$. We claim, that

$$
\lim _{k \rightarrow \infty} \frac{S_{n_{2 k+1}}(B)}{S_{n_{2 k+1}}}=0
$$

Indeed,

$$
\begin{aligned}
S_{n_{2 k+1}}(B) & \leq S_{n_{2 k+1}}\left(\left(E-P_{2 k+1}+P_{2 k}\right) A\right) \\
& =S_{n_{2 k+1}+\left(n_{2 k+1}-n_{2 k}\right)}-\left(S_{n_{2 k+1}}-S_{\left.n_{2 k}\right)}\right. \\
& \leq S_{2 n_{2 k+1}}+S_{n_{2 k}}-S_{n_{2 k+1}}
\end{aligned}
$$

which, by (3) and (4) proves our claim. So $B=F A$ satisfies condition (5) of Lemma 4. Similarly, the same can be proved for $B^{\prime}=(E-F) A=A-F A$. Assume now, that (6) is satisfied by $B=F A$. Then

$$
0=A-F A+\sum_{i=1}^{N+1}\left(-a_{i}\right) U_{i} A U_{i}^{*}+\sum_{j=1}^{M} K_{j} A\left(-L_{j}\right)
$$


where $a_{N+1}=1$ and $U_{N+1}=i d$. This means, that-although with different parameters $-B^{\prime}$ satisfies (6) too. So $\sum_{i=1}^{N} a_{i}=0$ and $\sum_{i=1}^{N+1}-a_{i}=0$, that is $a_{N+1}=0$, a contradiction. Consequently (6) can hold for $B=F A$ with no parameters, in other words $F A \notin \mathscr{J}(\mathscr{U}(A))$. But clearly $F A \in \mathscr{I}(\mathscr{A})$, so the proof of the theorem is complete.

TRACEABLE OPERATORS

Let $\mathscr{L}(A)$ be the Lie ideal in $\mathscr{B}(\mathscr{H})$ generated by $A \in \mathscr{B}(\mathscr{H})$. By [1, Theorem 1]

$$
\begin{gathered}
\mathscr{L}(A)=\operatorname{span}(\mathscr{U}(A))= \\
\left\{\sum_{i=1}^{N} a_{i} U_{i} A U_{i}^{*}: N \geq 0, a_{i} \in \mathbf{C}, U_{i}^{*}=U_{i}^{-1}(i=1,2, \ldots N)\right\} .
\end{gathered}
$$

Lemma 4 suggests the following

Definition. Let us say that an operator $A$ is traceable if any of the following three equivalent conditions hold:

(i) There exists a linear functional $f$ on $\mathscr{L}(A)$ such that $f\left(U A U^{*}\right)=1$ for every unitary operator $U$.

(ii) $\sum_{i=1}^{N} a_{i} U_{i} A U_{i}^{*}=0\left(U_{i}^{*}=U_{i}^{-1}\right)$ implies $\sum_{i=1}^{N} a_{i}=0$.

(iii) $0 \notin\left\{\sum_{i=1}^{N} \lambda_{i} U_{i} A U_{i}^{*}: \sum_{i=1}^{N} \lambda_{i}=1\right\}$.

For the implication (ii) $\Rightarrow$ (i) we (well-)define $f$ by $f\left(\sum_{i=1}^{N} a_{i} U_{i} A U_{i}^{*}\right)=$ $\sum_{i=1}^{N} a_{i}$. If $A$ is traceable, we put $\mathscr{L}_{0}(A)=\operatorname{ker} f . \mathscr{L}_{0}(A)$ is a Lie ideal in $\mathscr{B}(\mathscr{H})$. If $A$ is as in the theorem, then $A$ is traceable even in the stronger sense that $f$ can be extended to a unitary invariant linear functional $\hat{f}$ on $\mathscr{J}(\mathscr{U}(A))$ so that $\mathscr{J}\left(\mathscr{L}_{0}(A)\right) \subset \operatorname{ker} \hat{f}$. Assume furthermore that $A=A^{\prime} \oplus 0 \in \mathscr{B}(\mathscr{H} \oplus \mathscr{H})$. Then $A^{\prime} \oplus\left(-A^{\prime}\right) \in \mathscr{L}_{0}(A)$, so $A \in \mathscr{I}\left(\mathscr{L}_{0}(A)\right)$ but clearly $A \notin \mathscr{J}\left(\mathscr{L}_{0}(A)\right)$, i.e. Lemma 4 itself gives a counterexample different from the first one.

It is clear that if the image of $A$ in the Calkin algebra is a nonzero constant, then $A$ is traceable. On the other hand, if the image of $A$ is not a constant in the Calkin algebra, then $A$ is not traceable. To see this, suppose that $f$ is a unitary invariant linear functional on $\mathscr{L}(A)$. By a theorem of Topping (see [1, Corollary 1]) $\mathscr{L}(A)=\mathscr{B}(\mathscr{L})=\mathscr{L}(\mathscr{P})$, where $\mathscr{P}$ is an orthogonal projection with $\operatorname{dim} \operatorname{ker} P=\operatorname{dim} \operatorname{ran} P$. But $P=P_{1}+P_{2}$, where $P_{1}$ and $P_{2}$ are unitary equivalent to $P$. So $f(P)=2 f(P)=0$, which implies $f \equiv 0$ on $\mathscr{B}(\mathscr{H})=\mathscr{L}(A)$. This shows that $A$ is not traceable. So,-assuming $\mathscr{H}$ is separable-among the noncompact operators we can identify the traceable ones. But in the case of compact operators the question seems more difficult. It is clear that if $K \in \mathscr{K}(\mathscr{H})$ then $K \oplus(-K)$ is not traceable, and that a trace-class operator with nonzero trace is traceable. The theorem of this paper 
gives an example of a traceable operator outside of trace-class. To see that even $0 \leq A \neq 0$ does not imply traceability, consider the following example:

$$
\begin{aligned}
& 2^{-1} \operatorname{diag}\left(1,0,0,2^{-1}, 2^{-1}, 0,0,4^{-1}, 4^{-1}, 4^{-1}, 4^{-1}, 0,0,0,0,0,0,0,0, \ldots\right) \oplus 0 \\
& +2^{-1} \operatorname{diag}\left(2^{-1}, 1,0,0,0,2^{-1}, 0,0,0,0,0,4^{-1}, 4^{-1}, 4^{-1}, 4^{-1}, 0,0,0,0, \ldots\right) \oplus 0 \\
& +2^{-1} \operatorname{diag}\left(2^{-1}, 0,1,0,0,0,2^{-1}, 0,0,0,0,0,0,0,0,4^{-1}, 4^{-1}, 4^{-1}, 4^{-1}, \ldots\right) \oplus 0 \\
& \quad=\operatorname{diag}\left(1,2^{-1}, 2^{-1}, 4^{-1}, 4^{-1}, 4^{-1}, 4^{-1}, 8^{-1}, 8^{-1}, 8^{-1}, 8^{-1}, 8^{-1}, 8^{-1}, 8^{-1}, \ldots\right) \oplus 0
\end{aligned}
$$

It would be interesting to know what the traceable compact operators exactly are.

\section{REFERENCES}

1. C. K. Fong, C. R. Miers and A. R. Sourour, Lie and Jordan ideals of operators on a Hilbert space, Proc. Amer. Math. Soc. 84(1982), 516-520.

2. C. K. Fong and H. Radjavi, On ideals and Lie ideals of compact operators, Math. Ann. B 262. H 1 (1983), 23-28.

3. C. K. Fong and G. J. Murphy, Ideals and Lie ideals of operators, Acta Sci. Math. (Szeged) 51(1987), 441-456.

4. I. C. Gohberg and M. G. Krein, Introduction to the theory of nonselfadjoint operators, Transl. Amer. Math. Soc., 18, Providence, RI, 1969.

Bolyai Institute, University of Szeged, Aradi Vértanuk, Tere 1. 6720 Hungary 